\title{
WALKING DROPLETS THROUGH THE LENS OF DYNAMICAL SYSTEMS
}

\author{
AMINUR RAHMAN ${ }^{*}, 1$ AND DENIS BLACKMORE ${ }^{2}$
}

\begin{abstract}
Over the past decade the study of fluidic droplets bouncing and skipping (or "walking") on a vibrating fluid bath has gone from an interesting experiment to a vibrant research field. The field exhibits challenging fluids problems, potential connections with quantum mechanics, and complex nonlinear dynamics. We detail advancements in the field of walking droplets through the lens of Dynamical Systems Theory, and outline questions that can be answered using dynamical systems analysis. The article begins by discussing the history of the fluidic experiments and their resemblance to quantum experiments. With this physics backdrop, we paint a portrait of the complex nonlinear dynamics present in physical models of various walking droplet systems. Naturally, these investigations lead to even more questions, and some unsolved problems that are bound to benefit from rigorous Dynamical Systems Analysis are outlined.
\end{abstract}

\section{INTRODUCTION}

Dynamical Systems Theory is often used to analyze complex phenomena that arise from the interaction of simpler components. One problem that comes to mind is the " $n$-body" problem. Solving the 2-body problem is very simple as shown by Newton [1]. However, as shown by Poincaré [2,3], adding even a small third body makes the problem quite complex. Similarly, with walking droplets, even the simplest reduction: a ball bouncing on a table, exhibits chaotic behavior [4].

It has been known since the 1970s that liquid droplets can float and bounce on a vertically sinusoidally accelerated liquid bath [5]. Three decades later, Couder and co-workers discovered that increasing the acceleration on the bath causes the droplet to move horizontally in addition to bouncing $[6,7]$, and thus are dubbed walking droplets (or walkers). Incredibly, they had created a classical system where the particle-like object (the droplet) generated waves, which then propelled the droplet. This was reminiscent of Pilot-wave Theory in Quantum Mechanics, where a wave guides the particle, thereby determining the statistics induced from particle trajectories. The similarities between walking droplets (or hydrodynamic pilot-waves) and Pilot-wave Theory of Quantum Mechanics, led to extensive research in using walking droplets to conduct Quantum-like experiments $[8,9]$. While some experiments captured much of the statistics observed in quantum systems, others did not. As with most complex systems, mathematical models play a pivotal role in analyzing walking droplets and making predictions about their behavior. The models provide insight into the

Key words and phrases. Dynamical Systems; Walking Droplets; Nonlinear Dynamics; Chaos.

${ }^{*}$ Corresponding Author (arahman2@uw.edu).

${ }^{1}$ Department of Applied Mathematics, University of Washington, Seattle, WA 98195.

${ }^{3}$ Department of Mathematical Sciences, New Jersey Institute of Technology, Newark, NJ 07102. 
possible dynamics that have yet to be observed in experiments. Rigorous dynamical systems analysis can aid experimentalists in fine-tuning the parameters to achieve the desired statistics of particle trajectories [10].

There is a rich history of scientific advances through the use of Dynamical Systems Theory. Sometimes the scientific phenomena inform the theory and other times the theory corrects our understanding of the phenomena. This is evident in the works of Poincaré $[2,3]$ where he initially had a particular understanding of the 3-body problem, which worked well for a short period of time, but as he delved further into the analysis he realized that his understanding needed to be modified. Dynamical Systems Theory has the potential to play a similar role in the walking droplets phenomenon. This potential has already manifested itself in several analytically rigorous studies, which will be discussed in this review.

The remainder of this article is organized as follows: In Sec. 2, some background on relevant quantum mechanical phenomena and interpretations are given. Section 3 takes us into the experimental setups that lead to promising analogies with Quantum Mechanics. Then we discuss the fluid dynamics models in Sec. 4. Finally in Sec. 5 and 6, we arrive at the main topic of this review: Dynamical Systems models and interesting results arising from those models. To conclude, in Sec. 7 we outline some unsolved problems that are ripe for rigorous Dynamical Systems analysis.

\section{Quantum Mechanics}

Since the nascence of Quantum Mechanics (QM), much of the controversy has been centered around its philosophical implications [11, 12]. In recent decades it has been shown to be one of the most predictive theories of Physics. Indeed, it is true that the mathematical foundations of QM are strong. However, the original controversy of its interpretation lingers. Some may question the merit in attempting to shed light on the subtleties of the quantum world. Since the predictions of QM are so precise, why should we not use it to just make measurements and leave the mysterious statistical world underneath undisturbed? We recall that the ancient Greeks had the ability to measure the location of the planets and stars in the night sky with remarkable precision. Yet, it was not until Kepler's laws of planetary motion and Newton's laws of gravity that we had both quantitative predictions and a correct qualitative description of the motion of celestial bodies. Thus, the walking droplets system, due to its similarity to the pilot-wave interpretation $[13,14,15,16,17,18]$, is an intriguing addition to this debate. Specifically, the walking droplets system is more analgous to de Broglie's "double-solution" theory [15] than Bohm's use of a stochastic field $[17,18,19,20,21,8]$. These and other connections to realist models of quantum dynamics have been explored thoroughly in review articles by Bush [8, 9] and Bush and Oza [22].

Unlike classical phenomena, quantum phenomena cannot yet be observed directly. However, this dilemma is nothing new in science. A major stumbling block to observing the exact dynamics of quantum particles is in our inability to precisely measure both momentum and position. One explanation could be that quantum particles are inherently Statistical and do not have definite properties until they are measured. Similarly, the ancient Greeks developed interpretations based on what they observed in the night sky. Newton and Huygens, on the other hand, did have access to data from telescopes, but these devices did not completely uncover the realities of planetary motion as our modern day satellites do. This necessitated analogies to bridge the gap between the available measurements and their unobservable qualitative description. They realized that a similar phenomenon would be a ball at the end of 
a string with a radially inward tensile force. From Newton's writings, it seems that this analogy aided him in developing his theory of gravitation centuries before high resolution observations of the motion of celestial bodies was possible.

\section{WALKing Droplets}

In the early days of QM, de Broglie and Bohm championed the interpretation where a pilot-wave governs the motion of a quantum particle $[13,17,18]$. In recent decades we finally have a directly observable macroscale analog of a pilot wave. Consider a table that is forced sinusoidally in the vertical direction creating a vibrating fluid bath (Fig. 1)[23]. Suppose that the table is forced with an acceleration of

$$
f(t)=\gamma \sin \omega t ; \quad \gamma=A_{0} \omega^{2} .
$$

The surface of the bath is initially flat. A droplet is dropped onto the bath resulting in

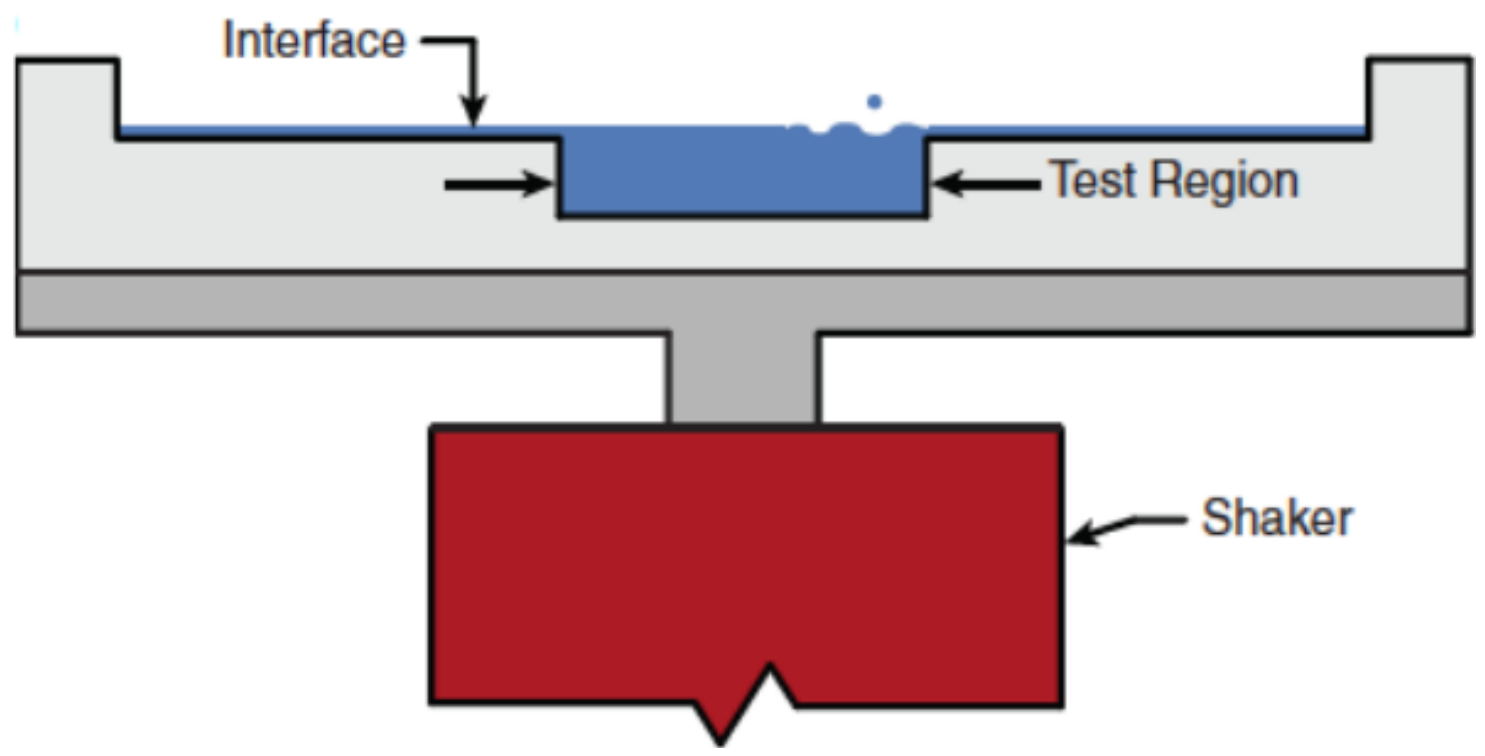

FiguRE 1. Experimental setup to produce walking droplets [24].

excitations to the initially flat wave field. Also, due to the surface tension of the droplet and the bath, the droplet is propelled upward instead of coalescing with the bath. A small excitation (small value of $\gamma$ ) would create a shallow crater in the bath that disappears when the droplet is in the air, and therefore the droplet just bounces. A larger excitation (large value of $\gamma$ ) creates a deeper crater that persists even after the droplet returns, and now the droplet can interact with the sides of the crater to be propelled opposite to the slope at that point. The subsequent impacts of the droplet with the bath contribute to the total wave field. Now we have a particle (the droplet, which we call a walker) being propelled by the waves that it creates; i.e., a pilot wave.

Since the seminal works of Couder and co-workers $[6,7,25,26]$, there have been many articles on fine-tuning droplet creation and the experimental setup [27, 28, 29]. In addition, it has since been shown that the air flow in the laboratory contributes to the statistics observed in the system, and therefore more recent experiments enclose the system to isolate the walkers from air $[30,31]$. However, for the purposes of this brief review, we are more 
interested in documenting the experiments that produce dynamics indicative of nonlinear and chaotic behavior.

Walking was discovered by increasing the acceleration on the fluid bath; i.e., increasing the value of $\gamma$. There have been extensive studies on the effects of bath acceleration [32, 26, 33, $34,35,36]$. If the bath acceleration is greater than a critical value $\gamma_{F}$, then standing waves called Faraday waves appear on the surface, and $\gamma_{F}$ is called the Faraday wave threshold [37]. Most often experiments are conducted below, but near the Faraday wave threshold, however there are studies of walking above the threshold, which will be discussed in subsequent sections. From a dynamical systems point of view, the transition from bouncing to walking is particularly interesting due to the bifurcations the system undergoes [33, 34, 38, 39]. While there is plenty of evidence for the observed bifurcations, many have yet to be rigorously proven, which creates a singular opportunity for dynamical systems analysts. It is common for the fluid dynamics models to be quite complex and not amenable to rigorous dynamical systems analysis so model reduction is often employed.

Although there are significant challenges to reproducing quantum statistics for experiments such as the single and double slit phenomena [30], corrals and rotating frames produce quite convincing quantum analogs. Fort et al. and Harris and Bush showed that a droplet in a rotating frame will tend towards quantized orbits [40, 41]. Due to the Coriolis effect on a mass in a rotating frame being analogous to the Lorenz force on charge in a uniform magnetic field [8], further analysis by Oza et al. [42, 43, 44] and Eddi et al. [45] solidified the analogy of walkers and quantum effects such as Zeeman splitting. Perhaps the most visually compelling example of an analog of a quantum experiment comes from the corral experiments. In the quantum corral experiment, first conducted by Crommie et al. [46], a ring of iron atoms are arranged on top of a copper surface causing the surface to form a wave within the boundaries of the ring. In the hydrodynamic corral experiments of Harris and Bush [23], they confine a droplet in a circular geometry, and amazingly the droplet produces a position distribution (Fig. 2) very similar to the wave produced in the quantum corral. Later studies by Cristea-Platon, Sáenz, and Bush also produced statistics similar to other quantum experiments [31, 47, 48].

Despite the promising experiments in confined geometries, confinement leads to complex boundary interactions, which have proved to be extremely challenging as shown by Pucci et al. [49, 50]. Simpler quasi-1-dimensional geometries, such as those studied by Filoux et al. [51, 52], are able to confine a droplet without the boundary having a significant impact on the position of the droplet. These types of confined geometries also naturally allow for dynamical systems models, which are discussed in more detail in Sec. 5.

\section{Fluid Dynamics Models}

To develop fluid dynamics models of the walker system we need to model the flight of the droplet in the air, the waves on the fluid bath, and the interaction between the droplet and the waves. Perhaps the simplest form of a particle interacting with a sinusoidally forced surface, which was investigated by Holmes, appears in the form of a ball bouncing on a vibrating table [4]. The system that Holmes studied can be modeled completely using kinematics. A similar hydrodynamic example was studied by Gilet and Bush where they bounce a droplet on a soap film [53, 54]. These ideas were used by Moláček and Bush to develop extremely detailed hydrodynamic models of droplets interacting with a vibrating fluid bath $[33,34]$. While these models captured a significant amount of the physics, they are 

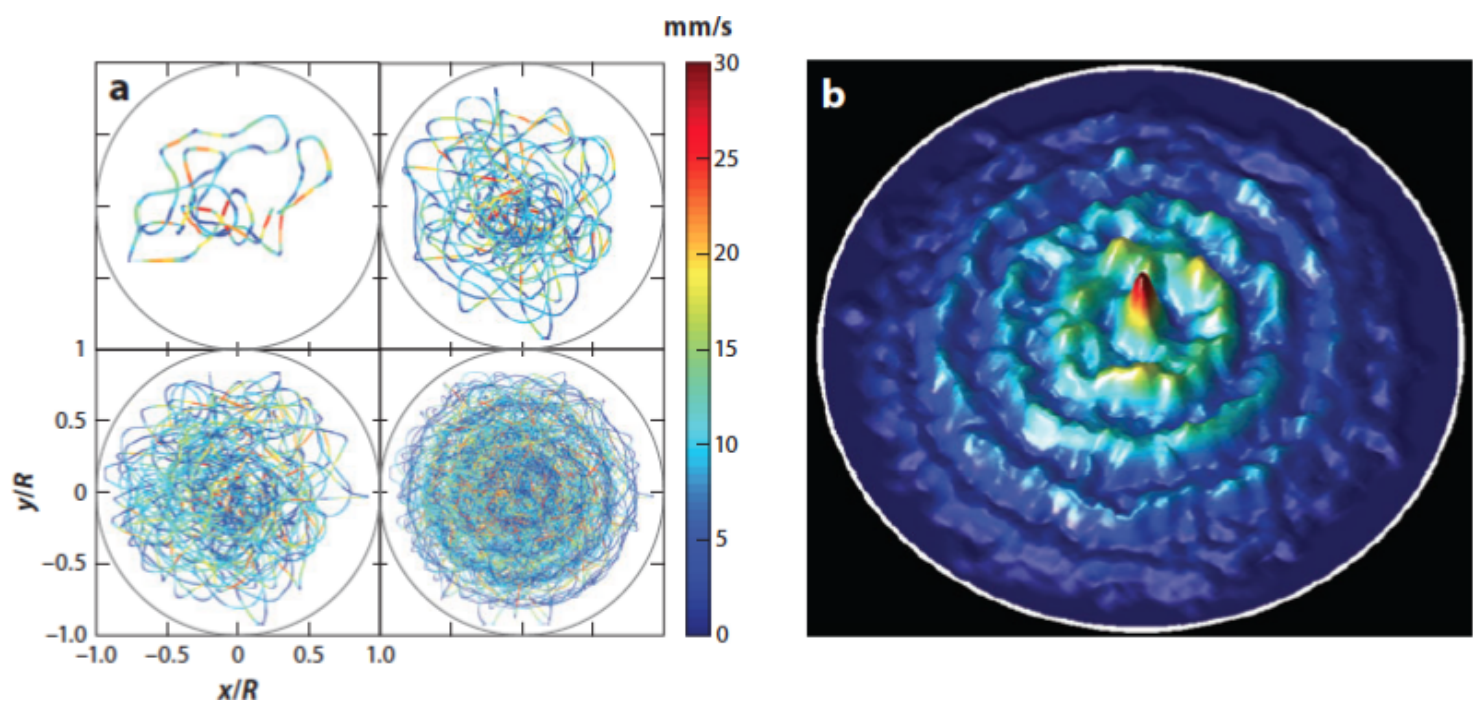

FiguRE 2. Distribution of walker location preference from the hydrodynamic corral experiments of Harris and Bush [23, 24].

also computationally expensive to simulate. Further, the bifurcations and exotic dynamical behavior is obfuscated by the various types of equations coupled to produce the model. For the purposes of this brief review, we are interested in outlining the dynamical systems results arising from the walking droplets system.

Much of the present bifurcation and chaotic behavior for bouncing can be inferred from the studies of Holmes [4] and Gilet and Bush [53, 54]. When the droplet goes from the bouncing to the walking regime, it undergoes period-doubling bifurcations, which were first observed in the experimental study of Moláček and Bush [33, 34]. In order to truly analyze the dynamics, we must make some simplifying assumptions. Oza et al. posited that the excitation from an impact travels fast enough through the bath before the subsequent impact that the droplet feels the same effect as it would if the excitation was instaneously a Bessel function [55]. This was dubbed the stroboscopic model since we are not interested in what happens between bounces.

For the stroboscopic model of Oza et al. [55], let us consider a case when the droplet creates a single impact at $x_{0}$ and then is taken away. In the following derivations we will assume the variables have been nondimensionalized, and all parameters have been reduced as much as possible. This single impact on the flat bath creates an excitation that takes the shape of a Bessel function of the first kind centered at and symmetric about $x_{0}, J_{0}\left(\left|x-x_{0}\right|\right)$ $[55,56]$. Since the system has natural damping due to the viscosity of the fluid, the excitation will decay exponentially over time, $t$, and hence the excitation becomes

$$
y=h_{0}(x, t)=J_{0}\left(\omega\left|x-x_{0}\right|\right) e^{-t} .
$$

In reality, the droplet falls back down after some time. Then the bath propels the droplet in a direction opposite to the gradient of the wavefield. The impact also contributes a new excitation to the wavefield, which is added to the old one to produce the new wavefield. All of the excitations are summed up to produce the total wavefield, $H(x, t)$. After each impact it is assumed that that droplet behaves like a projectile, and therefore the position, $x$, of the droplet is modeled as

$$
m \ddot{x}+D \dot{x}=-m g \nabla H(x, t)
$$


where $\mathrm{D}$ is the drag coefficient on the droplet [55]. For a much more detailed derivation, the reader is referred to the works of Oza et al. [55] and Moláček and Bush [33, 34].

While the stroboscopic model provides a natural transition to dynamical systems models, other types of models have numerically shown to exhibit intersting dynamical behavior. For example, the works of Milewski et al. [57], Durey et al. [58, 59], Galeano-Rios et al. [60], Faria [61], and others employ a quasi-potential wave model with various forms of droplet motion. This was recently extended to the experiments of Valani et al. on superwalkers [62] by Galeano-Rios et al. [63] where the bath is forced at two driving frequencies. Although the original stroboscopic model is designed for the high memory regime, and indeed most of the quantum-like statistics arise within this regime, interesting behavior is also observed in the low memory regime. In fact, some of the first such models only considered a single previous impact [26], similar to the assumption made earlier in this Section. In the low memory regime there are also electrodynamics analogs such as a boost to the walker's effective mass provided by the wavefield as shown by Bush et al. [64] and Labousse and Perrard [65].

One major challenge in modeling walking droplets are boundary effects. These are still not well understood, but there has been significant effort from both the experimental, which was discussed in the previous section, and theoretical sides, in resolving the dynamics at boundaries. If specular reflection is assumed, then the droplet dynamics is similar to that of billiards [66]: an observation that may have been useful. However, it was shown that this is not the case [49], and therefore boundary interactions need to be studied in more detail.

\section{Dynamical Systems Models}

In this section we shall present a concise but rather complete description of most of the leading mathematical models of walking single droplet phenomena, with a focus on those that promise the most fertile ground for further rigorous dynamical systems analysis. We begin with continuous dynamical systems (differential equations) models, which is only natural and fitting, since they represent the first realistic, hydrodynamic science based efforts to describe and predict the motion of walking droplets. Then, we turn our attention to discrete dynamical systems (difference equations) models, which for the most part have been derived formally or in an informed intuitive manner from continuous formulations.

5.1. Continuous dynamical models. Starting with pioneering efforts based on the fundamentals of physics complemented by observation of experiments, in Protière et al. [26], Eddi et al. [56] and Fort et al. [40], dynamical systems based modeling of walking droplet (pilot-wave) phenomena has come a long way. More precisely, building upon the pioneering work with the aid of several realistic simplifying assumptions, Moláček \& Bush [33, 34] and Oza et al. [55] developed a delay dynamical system model of the following form for the horizontal and vertical motion of a single walking droplet:

$$
m \ddot{\boldsymbol{r}}+D \dot{\boldsymbol{r}}=-m g \nabla h\left(\boldsymbol{r}, \boldsymbol{r}_{(t)}, t\right)-F(\boldsymbol{r}), \ddot{z}+m[g+\gamma(t)]=Z(\bar{z}, \dot{\bar{z}} ; k, b),
$$

where $:=d / d t, \boldsymbol{r}$ is a point in the region $\Omega \subset \mathbb{R}^{q}, q=1,2$, representing the oscillating floor of the container of the liquid bath, $z \in \mathbb{R}$ is the vertical position of the bottom of the droplet with respect to the undeformed initial surface of the bath, $m$ is the mass of the droplet, $g$ is the gravitational acceleration, and $\gamma(t)$ is the vertical acceleration of the vibrating bath. Here $F(\boldsymbol{r})$ is a possible horizontal force such as occurs in a rotating corral, $\bar{z}:=z-h\left(\boldsymbol{r}, \boldsymbol{r}_{(t)}, t\right)$, $\nabla$ is the gradient operator with respect to $\boldsymbol{r}, k$ and $b$ are, respectively, spring and damping constant parameters, $Z(\bar{z}, \dot{\bar{z}} ; k, b)$ is a smooth approximation of $H(-\bar{z}) \max (-k \bar{z}-b \dot{\bar{z}}, 0)$, 
with $H$ the Heaviside step function. Also, $h\left(\boldsymbol{r}, \boldsymbol{r}_{(t)}, t\right)$ is the wave field height of the oscillating (free) surface of the bath, which is a function of $\boldsymbol{r}, t$ and the delay $\boldsymbol{r}_{(t)}$ which denotes the function $\boldsymbol{r}:[0, \infty) \rightarrow \mathbb{R}^{q}$ restricted to $[0, t]$, which we expect to be smooth. It is precisely in $h$ that the delay occurs, which is also referred to as path-memory.

The above system involves delay differential equations, which lead to infinite-dimensional dynamical systems that tend to be difficult to analyze (see [67, 68, 69]). To be a bit more specific, the phase space vector and infinite-dimensional dynamical system, respectively, take the form $u:=(\boldsymbol{r}, \dot{\boldsymbol{r}}, z, \dot{z}, h), \dot{u}=\mathcal{F}(u)$ on the infinite-dimensional manifold $\mathcal{M}:=\mathbb{R}^{2(l+1)} \times C^{1}\left([0, \infty), \mathbb{R}^{l}\right)$, where $C^{1}\left([0, \infty), \mathbb{R}^{l}\right)$ denotes the continuously differentiable functions mapping the interval $[0, \infty)$ into $\mathbb{R}^{l}$.

Fortunately, a justifiable additional assumption enables the delay differential equations to be recast as the following integro-differential equation (IDE) system:

$$
\begin{aligned}
m \ddot{\boldsymbol{r}}+D \dot{\boldsymbol{r}} & =-m g \nabla h(\boldsymbol{r}, t)-F(\boldsymbol{r}, \dot{\boldsymbol{r}}) \\
\ddot{z} & =-m[g+\gamma(t)]+Z(\bar{z}, \dot{\bar{z}} ; k, b)
\end{aligned}
$$

where

$$
h(\boldsymbol{r}, t):=\frac{A}{T_{F}} \int_{-\infty}^{t} J_{0}\left(k_{F}|\boldsymbol{r}-\boldsymbol{r}(\tau)|\right) e^{-(t-\tau) / T_{F} M e} d \tau,
$$

with the understanding that $\boldsymbol{r}(\tau):=\boldsymbol{r}(0)$ for $\tau \leq 0$. In the above, $A$ is the initial amplitude of the generated waves, $T_{F}$ is the Faraday time between bounces, $J_{0}$ is the zeroth order Bessel function of the first kind, $k_{F}:=2 \pi / \lambda_{F}$ is the wave number corresponding to the least stable Faraday wave with wavelength $\lambda_{F}$ and $M e$ is the memory parameter that determines the damping speed of prior bounce generated waves. The first equation of (4) is usually referred to as the trajectory equation, which determines the horizontal dynamics, while the second equation is said to describe the vertical dynamics. It should be noted that the vertical dynamics (4b) can be modeled in several ways, often as a linear or nonlinear spring $[53,54,34,60]$.

The system (4) with (5) still represents an infinite-dimensional dynamical system (in fact, typically $\aleph_{0}$-dimensional) of the form

$$
\dot{u}=\mathfrak{F}(u ; \kappa)
$$

with $u=(\boldsymbol{r}, \dot{\boldsymbol{r}}, z, \dot{z}, h)$ on the infinite-dimensional manifold $\mathcal{M}:=\mathbb{R}^{2(l+1)} \times C^{1}\left([0, \infty), \mathbb{R}^{l}\right)$ and $\kappa$ represents the vector of physical parameters associated with the system, but it is easier to study via numerical simulation and appears to be more amenable to extensive dynamical systems analysis.

Penultimately, we mention the quasi-potential formulation of Milewski et al.[57] not only because it represents a more complete incorporation of fundamental fluid dynamics principles than most other extant models, but it also can be reduced to a form with a corresponding discrete dynamical system, which we shall briefly describe in the next subsection. The model is for an infinite domain and the wave dynamics can be conveniently expressed in cylindrical coordinates $(r, \theta, z)$, where one is able, by making very reasonable assumptions, to describe the model in terms of a velocity potential $\phi(r, \theta, z, t)$ and a perturbation $\eta(r, \theta, t))$ satisfying the following evolution equations and auxiliary conditions:

$$
\begin{aligned}
& \phi_{t}=-g_{L}(t)+2 \nu \Delta_{H} \phi+(\sigma / \rho) \Delta_{H} \eta-P(r, \theta, t), z=0 \\
& \eta_{t}=\phi_{t}+2 \nu \Delta_{H} \eta, z=0
\end{aligned}
$$


and

$$
\Delta \phi=0, \text { for } z \leq 0, \nabla \phi \rightarrow 0 \text { and } \eta \rightarrow 0 \text { as } r^{2}+z^{2} \rightarrow \infty,
$$

where $g_{L}$ is the effective gravity in the vibrating frame, $\sigma$ is the tension, $\rho$ is the constant density and $\nu$ the kinematic viscosity, respectively, of the incompressible fluid bath, $\Delta_{H} \phi:=$ $r^{-1}\left(r \phi_{r}\right)_{r}+r^{-2} \phi_{\theta \theta}$ is the horizontal Laplacian, $\Delta \phi:=r^{-1}\left(r \phi_{r}\right)_{r}+r^{-2} \phi_{\theta \theta}+\phi_{z z}$ is the Laplacian, and $P$ is the droplet impact pressure on the bath surface. These equations are then combined with the droplet dynamics, which may be taken as essentially the same as in second equation of (4), to obtain an infinite-dimensional dynamical system of the form

$$
\dot{w}=\mathfrak{M}(w ; \kappa)
$$

for $w:=(\phi, \eta, \boldsymbol{r}, \dot{\boldsymbol{r}}, z, \dot{z})$ on an infinite-dimensional manifold of the form $\mathcal{M}_{M}:=\mathcal{M}_{\phi} \times \mathcal{M}_{\eta} \times$ $\mathbb{R}^{6}$, where $\mathcal{M}_{\phi}$ comprises the functions $\phi$ satisfying (8) for all $t \geq 0$ and $\mathcal{M}_{\eta}$ consists of all $C^{2}$ functions $\eta$ satisfying (8) for all $t \geq 0$. As one might expect, the extra attention to physical details in the dynamical system (9) makes it more difficult to simulate and analyze than (6). A reduced form of the system that is solvable numerically at a much smaller computational cost was developed by Durey and Milewski [58], and extended by Faria [61]. Similarly, the stroboscopic model was reduced to a Lorenz-like dynamical system in an investigation by Durey [70]. Finally, we call attention to the interesting quasi-potential model of Galeano-Rios et al. [60], which can also be extended to model superwalkers [63].

Remark 1. It is interesting to observe that all the dynamical systems models considered in this section (and most of the others not treated here) comprise two coupled components: a (horizontal) trajectory equation; and a (vertical) droplet dynamics equation. Moreover, the coupling is essentially one-way, inasmuch as it is possible to solve for the trajectory independently of the droplet motion owing to the fact that this entails the calculation of the wave field on the surface of the liquid bath, but determination of the droplet dynamics requires the solution of the trajectory equation because it involves the wave field. This probably explains why droplet dynamics often played a secondary role early on in hydrodynamic pilot-wave research. In recent years, droplet dynamics has been receiving increasingly more attention necessitating the use of Dynamical Systems Theory.

5.2. Discrete dynamical system models. Continuous dynamical systems such as those treated above can often, via a variety of methods that include simplifying assumptions, reduction techniques, and experimentally-based inferences, be used to obtain corresponding discrete dynamical systems models, which are invariably easier to simulate and analyze than the systems from which they are derived. Now given any continuous dynamical system, finite or infinite dimensional, of the form $\dot{u}=G(u)$, one can always obtain a discrete dynamical approximation via numerical integration, where the accuracy depends on the choice of the method and the increments chosen for the variables. Such methods include complete finite difference or finite element approaches and semidiscrete schemes in which the space variables are handled via finite difference, finite element, spectral, or pseudo-spectral methods that produce a system of ordinary differential equations that are resolved using one-step techniques such as Runge-Kutta or multistep methods such as those of the Adams-Moulton predictor-corrector type, or some variation of these (see [71]).

An example of a variant of the numerical integration schemes can be illustrated in outline using the system (4). As mentioned in Remark 1, it essentially suffices to show how the 
first (trajectory) IDE can be integrated numerically. First, we introduce the variable $\boldsymbol{w}:=$ $(\boldsymbol{r}, \boldsymbol{v}):=(\boldsymbol{r}, \dot{\boldsymbol{r}})$, whereupon the trajectory equation can be recast as

$$
\dot{\boldsymbol{w}}=\boldsymbol{\Phi}(\boldsymbol{w}, t ; \kappa):=\left(\boldsymbol{v},-\frac{1}{m}(D \boldsymbol{v}+F(\boldsymbol{r})-g \nabla h(\boldsymbol{r}, t))\right) .
$$

This equation can then be converted to a finite difference approximation using a standard one-step integrator such as the Runge-Kutta method, with the only wrinkle being that the integral has to be approximated by a standard numerical scheme. For another example - one of standard semidiscrete type - consider the system of partial differential equations (7), which for convenience we consider on a bounded domain with boundary conditions and harmonic constraint as in (8). Then the spatial requirements, including the harmonic constraint can be approximated by finite differences, after which one has a system of ODEs for the approximations, which could be solved by an Adams-Moulton predictor-corrector scheme.

A specific example similar to approaches sketched above was developed and studied by Shirokoff [66]. He essentially begins with the trajectory equation of (4), makes some plausible simplifying assumptions including periodic droplet impacts of period $T$, and then integrates over a period to obtain the following $\aleph_{0}$-dimensional difference equation system for the position and velocity at the $(n+1)^{\text {th }}$ impact:

$$
\left(\boldsymbol{y}_{n+1}, \boldsymbol{v}_{n+1}\right)=\left(\boldsymbol{y}_{n}+\boldsymbol{v}_{n}, v_{n}+R\left(v_{n}, y_{n}, y_{n-1}, \ldots y_{1} ; \kappa\right)\right),
$$

where $\boldsymbol{y}$ is the position of the droplet in the domain $\Omega \subset \mathbb{R}^{2}, \boldsymbol{v}$ is the velocity $\dot{\boldsymbol{y}}, \kappa$ is, as usual, the physical parameter vector and $R$ is a $C^{1}$ map. The infinite dimensionality of the above system makes even it difficult to simulate, so Shirokoff makes a further short memory assumption: namely, that the function $R$ above does not depend on $y_{k}$ for $k<$ $n-1$. Consequently, the discrete dynamical system (10) can be reduced to the following 8-dimensional model for $\boldsymbol{u}_{n}:=\left(\boldsymbol{y}_{n-1}, \boldsymbol{y}_{n}, \boldsymbol{v}_{n}\right)$ :

$$
\boldsymbol{u}_{n+1}=S\left(\boldsymbol{u}_{n} ; \kappa\right):=\left(\boldsymbol{y}_{n}, \boldsymbol{y}_{n}+\boldsymbol{v}_{n}, v_{n}+R\left(v_{n}, y_{n}, y_{n-1}, \ldots y_{1} ; \kappa\right)\right),
$$

which is much easier to analyze and simulate than (11).

Before describing what appears to be the simplest discrete dynamical system capable of exhibiting a good deal of the dynamics observed in experiments and simulations of more complicated mathematical models, we shall briefly mention a discrete dynamical model obtained from the system (9). Durey and Milewski [58] show how (9) can be reduced so that it can be formulated as a rather simple homogeneous system with jump conditions. This reformulation in essence represents the droplet-bath interactions as instantaneous, thereby enabling the system to be recast as a discrete dynamical system of the form

$$
\boldsymbol{\xi}_{n+1}=\mathcal{M}\left(\boldsymbol{\xi}_{n}, \kappa\right)
$$

where the $\boldsymbol{\xi}_{n}$ are $\aleph_{0}$-dimensional vectors containing droplet position information and wave field characterizations of the wave field of the the oscillating bath surface in term of coefficients of the corresponding Fourier-Bessel expansions. System (13) is indeed complicated, but can be approximated by finite-dimensional systems by truncating the Fourier-Bessel expansions of the wave fields.

For our final discrete dynamical system model for walking droplets, we consider the "toy" models of Gilet $[72,73]$, which he described as the simplest model that he thought capable of capturing a good deal of experimentally observed, confined wave-particle coupling dynamics. 
As it turns out, Gilet's toy models do indeed exhibit quite a few of the dynamical regimes even some of the most complex - observed in experiments and obtained from simulations of more physically complete dynamical systems representations of walking droplet phenomena.

We begin with Gilet's 1-dimensional bath model, where the domain is a finite interval, say $\Omega:=[\alpha, \beta]$, which can be considered as $[-\pi / 2,3 \pi / 2]$ by scaling. The domain can then be specified by the eigenfunctions of the 1-dimensional Laplace operator $\Delta_{1}:=d^{2} / d x^{2}$ with Dirichlet or Neumann boundary conditions. For example, in the latter case we note that the basis of eigenfunctions is $E_{N}(\Omega)=\{\sin (2 n+1) x: n \in \mathbb{Z}, n \geq 0\}$, where $\mathbb{Z}$ is the integers, and in what follows we define an eigenmode $\Psi$ for this and higher dimensional models to be a $C^{1}$ (Fourier) expansion in the basis of Laplacian eigenfunctions for the domain $\Omega$. Gilet's 1-dimensional bath model is a (vector) difference equation of the form

$$
\begin{aligned}
x_{n+1} & =x_{n}-C z_{n} \Psi^{\prime}\left(x_{n}\right) \\
z_{n+1} & =\mu\left[z_{n}+\Psi\left(x_{n}\right)\right],
\end{aligned}
$$

where $x_{n}$ is the position of the droplet along the bath, $C \in[0,1]$ represents the wave-particle coupling, $z_{n}$ is the wave field amplitude on the bath surface just prior to an impact, so it can be considered as defining the vertical dynamics of the droplet, $\Psi$ and $\Psi^{\prime}$ are, respectively, the (assumed) single eigenmode and its spatial derivative and $\mu \in[0,1]$ is the path-memory related damping factor. An illustration of the model is shown in Fig. 3. Owing to its

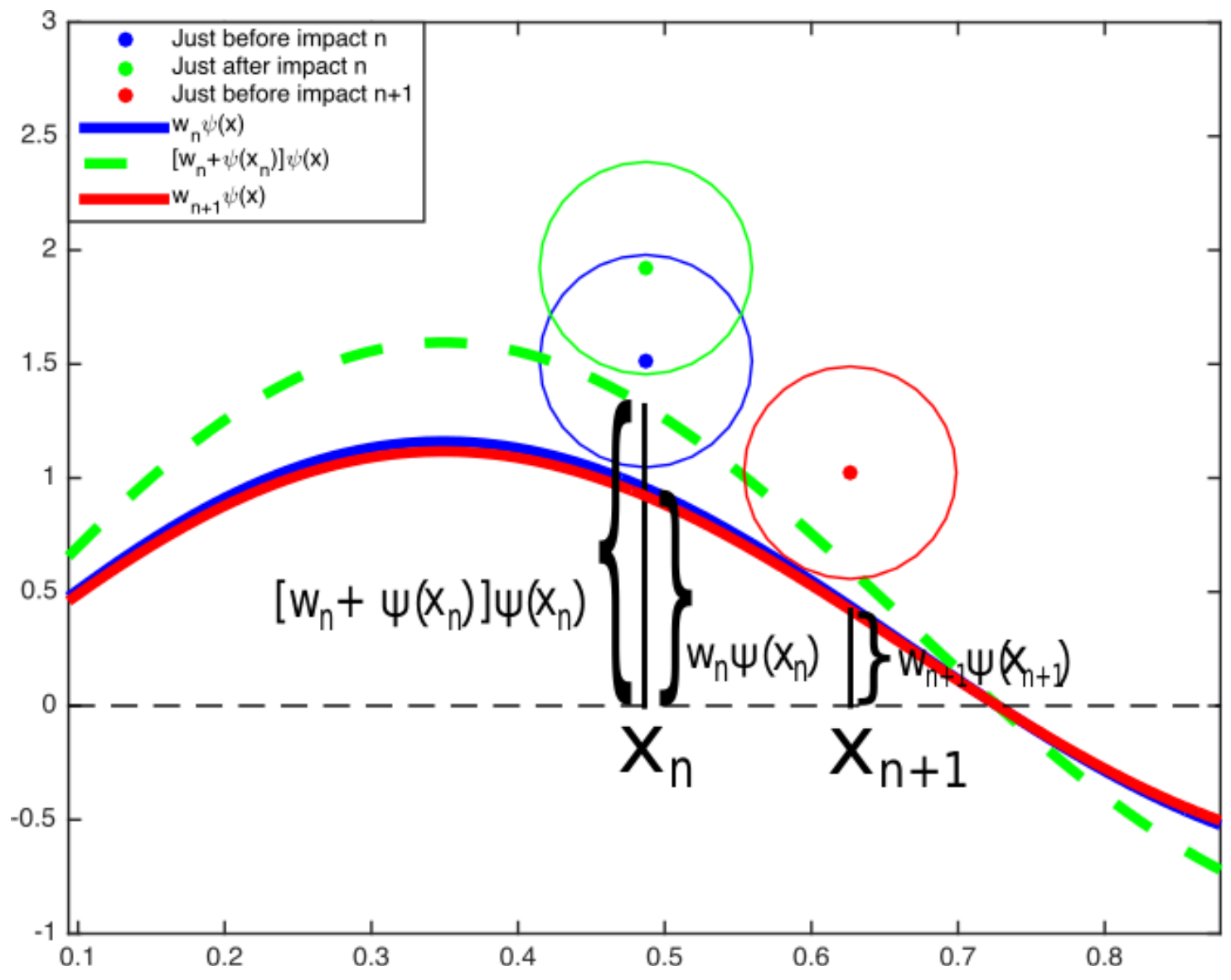

FiguRE 3. Illustration of the model (14) [74]. 
form and the periodicity of the eigenmode, one can conveniently extend the system (14) to the whole $x, z$-plane, so the dynamics can be represented by the iterates of the map $G_{1}: \mathbb{R}^{2} \rightarrow \mathbb{R}^{2}$ defined as

$$
G_{1}(x, z):=\left(x-C z \Psi^{\prime}(x), \mu[z+\Psi(x)]\right) .
$$

Not surprisingly, Gilet's 2-dimensional bath model for $\Omega \subset \mathbb{R}^{2}$, assuming a single eigenmode $\Psi$ with a $C^{1}$ expansions in terms of the Dirichlet or Neumann eigenfunctions on $\Omega$ of $\Delta:=\partial^{2} / \partial x^{2}+\partial^{2} / \partial y^{2}$, is a map $G_{2}: \mathbb{R}^{3} \rightarrow \mathbb{R}^{3}$ defined as

$$
G_{2}(x, y, z):=\left(x-C z \Psi_{x}(x, y), y-C z \Psi_{y}(x, y), \mu[z+\Psi(x, y)]\right),
$$

where the eigenmodes $\Psi$ would be represented in Fourier-Bessel series and Fourier-Mathieu series for circular and elliptic corrals, respectively. Clearly, Gilet's models are easy to simulate and they turn out to be comparatively amenable to rigorous dynamical systems analysis.

Another type of discrete dynamical model, developed by Rahman [75], models multiple walkers with a bath acceleration below the Faraday wave threshold (describing the experiments of Filoux et al. [51]) and a single walker with the bath acceleration above the Faraday wave threshold. For the single walker model, pitchfork and period doubling bifurcations, and chaos is observed and rigorously proved via Dynamical Systems Analysis. In this model, it is assumed that the velocity receives a "kick" at each impact, and therefore is similar to the Standard map [76, 77, 78]. The velocity becomes

$$
v_{n+1}=f\left(v_{n}, \theta_{n}\right)=C\left[v_{n}+K \sin \left(\omega\left(\theta_{n}-\theta_{n-1}\right)\right) e^{-\nu\left(\theta_{n}-\theta_{n-1}\right)^{2}}\right],
$$

where $v_{n}$ and $\theta_{n}$ are the velocity and positions of the single droplet after the $n^{\text {th }}$ impact. The nondimensional wavenumber is $\omega=2 \pi\left(R_{\text {in }}+D / 2\right) / \lambda_{F}$, where $\lambda_{F}$ is the Faraday wavelength and $R_{\text {in }}+D / 2$ are the averages of the inner and outer radii. The spatial damping of the kick strength is represented by the exponential term with a damping parameter $\nu \in \mathbb{R}^{+}$, which implicitly depends on $\lambda_{F}$ and the memory of the system. Then, just as with the Standard map, if $\theta_{n+1}=\theta_{n}+v_{n+1}$,

$$
f(v):=C\left[v+K \sin (\omega v) e^{-\nu v^{2}}\right] .
$$

The new 1-dimensional map preserved significant qualitative features of walkers on an annulus, and was well-suited for rigorous Dynamical Systems analysis.

\section{Dynamical Systems Results and Conjectures}

Now we shall encapsulate some of the more important dynamical systems related results obtained from studies of the mathematical models described in the preceding section, followed by some conjectures based upon the knowledge distilled from the investigations. The results shall be delineated in the context of modern dynamical systems theory[79, 80, 81, 82, 68, 83, 69, 84]. As we shall see, the complexities of most of the extant models for walking droplet dynamics have led to a preponderance of results being of the experimental or numerical simulation variety.

6.1. Results obtained from investigation of dynamical systems models. Using Section 5 as a guide, we begin with the continuous and end with the discrete dynamical systems models. Although the quantum analogs of walking droplet phenomena are fascinating, our focus shall be almost exclusively the dynamical systems related outcomes. 
6.1.1. Outcomes for continuous dynamical systems models. The early investigations of walking droplet phenomena produced a number of interesting dynamical systems related observations and conclusions. For example, Protière et al. [26] reported symmetry breaking, pitchfork, and period-doubling bifurcations based upon experimental observations and approximate solution of a relatively simple model for the trajectory dynamics. Several years later, employing experiments and a phenomenological model, Eddi et al. [56] also observed symmetry breaking and period-doubling in single droplet period trajectory dynamics as well as travelling wave dynamics and indications of transition to chaotic regimes. Fort et al. [40] investigated the motion of a single walking droplet in a rotating circular corral both experimentally and using a trajectory equation that might be considered a simplified version of that in the system (4). Experiments and numerical solution of their trajectory equation showed the existence of stable periodic (circular) trajectories occurring in discrete sets in quantum-like fashion. Orbital quantization and the emergence of quantum-like statistics were later considered by Perrard et al. [85], Harris and Bush [41], Bush [8], and Gilet [73].

More recent studies of continuous evolution mathematical models of walking droplet phenomena, which along with much of the material in this paper, have been very adroitly summarized in such papers as Bush [8, 9, 22] and Turton et al. [86], have uncovered many more results amounting to an extensive menagerie of dynamic possibilities. Many of the more contemporary investigations have been inspired by the seminal work of Moláček and Bush [33, 34] and Oza et al. [55]. The results of the investigations in [33, 34], which comprise both experimental and theoretical components, include a dynamical model with a trajectory equation that is essentially a simplified precursor of that in (4), together, ultimately, with a logarithmic spring vertical dynamics equation. Numerical integration of their model showed the existence of pitchfork bifurcations for a sufficiently large forcing acceleration $\gamma$ (or memory $\mathrm{Me}$ ) followed by period-doubling cascades leading to chaos as the parameter increased; outcomes that were in good agreement with experimental results. Oza et al. [55] devised the trajectory equation in (4), which has proven to be one of the most reliable qualitative and quantitative predictors of horizontal walking droplet dynamics. Using analysis and some numerics on their equation, they demonstrated the existence of a supercritical pitchfork bifurcation for sufficiently large $\gamma$ (or $M e$ ) that generates a rectilinear trajectory. Moreover, they showed that this trajectory tends to destabilize transversely with increasing $M e$, suggesting that analogous destabilization of more complicated orbits might lead to chaotic dynamics.

The theoretical developments of John Bush's group at MIT has been substantial [33, 34, 55]. For example, in an investigation combining experiments and theory based on the model in [34], Wind-Willassen et al. [35] found, by numerical integration of their model, that as $\gamma$ was increased, several period-doubling sequences were generated, and finally that chaotic regions appeared for sufficiently large parameter values. These outcomes turned out to be in rather good agreement with their experimental results, which supported the efficacy of the theoretical approach. There is also the rotating corral study of Oza et al. [42] focused on the trajectory equation of (4) with a Coriolis force $F:=2 m \boldsymbol{\omega} \times \dot{\boldsymbol{r}}$. They found, using both analytical and numerical tools, that as $M e$ increased, quantized stable circular orbits appeared, then tended to wobble and destabilize, followed by period-doubling that seemed to lead to chaotic regimes. In another related investigation, Oza et al. [43] studied the same trajectory equation for the horizontal dynamics, with an eye toward behavior resulting from increasing memory. Integrating the trajectory equation using the Adams-Bashforth 
method, they found that as $M e$ was increased beyond the wobbling circular orbit phase, the trajectories tended to drift into increasingly complicated periodic and quasiperiodic trajectories and ultimately chaotic attractors as $M e$ approached unity. The fact that the conclusions of these last two papers is in reasonably good agreement with the experimental results in Harris et al. [24] and Harris and Bush [41], lends considerable credence to their fidelity.

More recently, Tambasco et al. [87] used and Adams-Bashforth method to solve the trajectory equation of (4) with a Coriolis, Coulomb or simple harmonic potential force. They found that increasing $\gamma$ (or $M e$ ) in the cases of Coriolis and Coulomb forces produced a period-doubling cascade to chaos, while for a simple harmonic force the transition to chaos resembled the mechanism described in Newhouse et al. [88]. Kurianski et al. [89] also considered the horizontal dynamics described by (4) for a simple harmonic potential force. They solved the corresponding trajectory equation using a combination of an AdamsBashforth integrator and Simpson's rule for the integral. The solutions exhibited some fairly complicated periodic trajectories, including trefoils and lemniscates, as well as quasiperiodic trajectories for fairly low values of $\gamma$, which, as the forcing was increased, transitioned to chaotic regimes that appeared to be decomposable into unstable quasiperiodic states.

Perrard and Labousse [90] studied the horizontal dynamics described by the system (4) confined by a radial horizontal force of the form $F:=c \boldsymbol{r}$. By using an essentially spectral approach based on expanding the wave field (5) in a Fourier-Bessel series and truncating it after a large number of terms, they reduced the system to a finite-dimensional approximation comprising a system of ordinary differential equations (ODEs) in the position, velocity and series coefficients. They then integrated the system numerically and found the expected behavior; namely, initially stable periodic orbits that destabilized and initiated a perioddoubling cascade to chaos with increasing $M e$. Moreover, the dynamical behavior they found from their theoretical approach was in good agreement with their experimental results.

Using a similar spectral-type approach, Budanur and Fleury [91] analyzed the dynamics of a finite-dimensional approximation of the same trajectory equation, modulo some symmetry reductions, studied in [90]. They truncated the Fourier-Bessel expansion of the wave field to obtain a 55-dimensional system of ODEs, which they investigated numerically in considerable detail. As a result, they found the expected period-doubling route to chaos with increasing Me mentioned above and a good deal more, including symmetry breaking bifurcations, supercritical Neimark-Sacker bifurcations [92, 93] of certain Poincaré sections generating invariant 2-tori, supercritical Andronov-Hopf bifurcations, and what appear to be global chaotic strange attractors associated with merging bifurcations related to interactions of invariant submanifolds and stable manifolds of fixed points and periodic orbits. Similarly, Durey [70], observed Hopf bifurcations, homoclinic bifurcations, and chaos in their Lorenzlike model.

There is also the quasi-potential model numerical of Galeano-Rios et al. [60, 63] performed using a combination of finite differences and a semi-implicit Euler method that produces dynamics that are in very good agreement with the experimental and theoretical results in Wind-Willassen et al. [35] and some of the other dynamical findings mentioned above. They also investigated a new dynamical regime called superwalkers, which was first observed by Valani et al. [62], wherein the droplet bounces in place when the bath container is subject to a simple harmonic vertical motion, but tends to walk exceptionally fast for mixed harmonic oscillations. 
In summary then, investigations of the existing continuous dynamical systems models, conducted almost exclusively by numerical techniques, have revealed a rich array of dynamics that apparently range from simple pitchfork bifurcations to chaotic strange attractors.

6.1.2. Dynamical results from discrete models. Some very interesting dynamics have been found in the investigation of the discrete dynamical system models described above. For example, Shirokoff [66] employed numerical simulation to study the dynamics of the finitedimensional difference equation (11), or equivalently the map (12) for two types of domains: $\Omega=\mathbb{R}^{2}$ or $\Omega=Q$, a square region. He focused on the changes in the dynamics as parameters $F$ and $\mu$, representing the force of the oscillation and fluid viscosity, respectively, were varied. The simulations showed that in the case when $\Omega=\mathbb{R}^{2}$, increasing $F$ led to a pitchfork bifurcation followed by a period-doubling cascade to chaos as the parameter was increased and there was analogous dynamical behavior with respect to variation of $\mu$. For the case of a square geometry, Shirokoff found, not surprisingly, that the bifurcation behavior depended on the size of $Q$. For a large square, the orbits tended toward a quasiperiodic attractor or a nearly dense attractor for sufficiently large $F$. On the other hand, for a small square, a periodic or quasiperiodic attractor emerged as the force increased, followed by apparently chaotic regimes and ultimately very complicated attractors for large enough $F$ values.

Simulation of the discrete dynamical system model of Durey and Milewski [58] also revealed some interesting dynamical behavior. Investigation of their model for $\Omega=\mathbb{R}^{2}$ showed the existence of pitchfork bifurcations and indications of period-doubling cascades with an increasing force parameter, which we denote by $\mathcal{F}$ for simplicity. For the same domain, but with the addition of a radial force, they observed discrete quantization behavior in the form of separated invariant closed curves that are initially stable and destabilize with increasing $\mathcal{F}$ while seemingly giving birth to stable tori and giving indications of a transition to chaos. They also simulated their model for a pair of droplets, which showed some of the bifurcations observed in the single droplet case and several more very complex dynamic regimes.

In contradistinction to the state-of-the-art for continuous dynamical systems models for walking droplet phenomena, there has been some rigorous analysis of the dynamics. Simulation studies of (15) indicated the existence of supercritical Neimark-Sacker bifurcations $[92,93]$ and rather unusual transitions to chaos (apparently due to the propinquity of growing attracting invariant simple closed curves to stable manifolds of fixed points) with increasing $C$ and $\mu$, as well as indications of long-time behavior analogous to quantization. Gilet concluded that the probability density function (PDF) for the model is proportional to $1 /\left|\Psi^{\prime}(x)\right|$. Most of these results were proved or analytically verified in Rahman and Blackmore [74, 94], where it was proved that $G_{1}$ can have both supercritical and subcritical Neimark-Sacker bifurcations [92, 93] with respect to either $C$ or $\mu$. Moreover, a rigorous explanation was provided for the chaos inducing mechanism for $C$ sufficiently close to unity, which involves the interaction of the basin of attraction of an invariant closed curve attractor and the stable manifold of a hyperbolic fixed point. This mechanism, which actually generates a chaotic strange attractor, was abstracted in Rahman et al. [95] in the form of a paradigm called the $\sigma$-map (illustrated in Fig. 4).

For the Standard map-like model of Rahman [75], they rigorously prove the existence of pitchfork and period doubling bifurcations and chaotic orbits as the damping parameter, $C$, is increased in (18). The two bifurcations are proved by conducting normal form calculations similar to those in Kuznetsov [96]. Through simulations of the model, one may observe chaos in the short timescale as the droplet suddenly changes velocity, as shown in Fig. 5, 

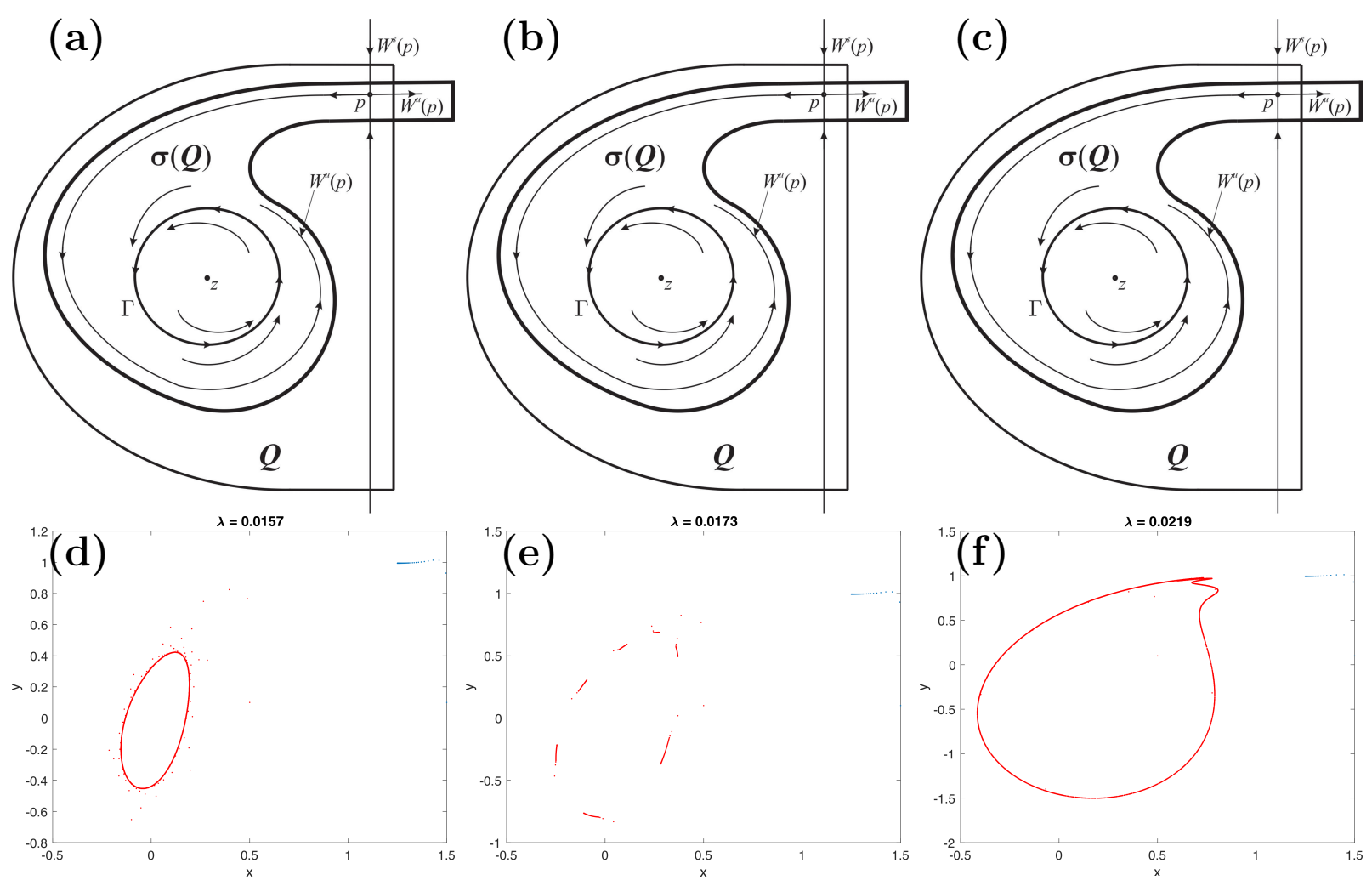

FiguRE 4. Illustration of the evolution of the $\sigma$-map through a homoclinictype bifurcation. Top row (a) - (c): diagrams of invariant manifolds as they evolve from left to right. Bottom row (d) - (f): Simulations of the model under the bifurcation respectively corresponding to the diagrams (a) - (c). (a), (d) Invariant manifolds are disjoint inducing regular dynamics. (b), (e) Once the invariant manifolds intersect tangentially, "blinking" behavior of the iterates begin; i.e., the iterates are not always dense within the invariant Jordan curve. (c), (f) The intersections are now transverse, and a stable chaotic strange attractor is generated.

in a seemingly random fashion. In the long timescale, the timeseries plot of Fig. 5, reveals complete disorder in the motion of the iterates. To definitively prove the existence of chaotic iterates, they rigorously show the existence of a 3-cycle (e.g. Fig. 5) and apply the theorem of Li and Yorke [97].

The model given by (16) assumes a single eigenmode, which is a special case of the one Gilet [73] considered for a circular corral that incorporated twelve dominant Neumann eigenmodes, each with its own damping factor $\mu_{k} \in[0,1]$. This leads to a 14-dimensional difference equation model equivalent to the discrete dynamics of a map of the form $\tilde{G}_{2}$ : $\Omega \times \mathbb{R}^{12} \rightarrow \Omega \times \mathbb{R}^{12}$ (which is essentially dynamically equivalent to $G_{2}$ ). The simulation studies in [73] showed that as the damping factors increases, the trajectory map, which is $\tilde{G}_{2}$ projected onto $\Omega$, exhibits stable periodic orbits that destabilize for sufficiently large $\mu_{k}$ via Neimark-Sacker cycle bifurcations [92, 93]. Also observed was that a further increase in the damping factors (with at least one of them slightly lower than unity) induces chaotic regimes. Moreover, calculations of radial positioning statistics for several simulations showed 

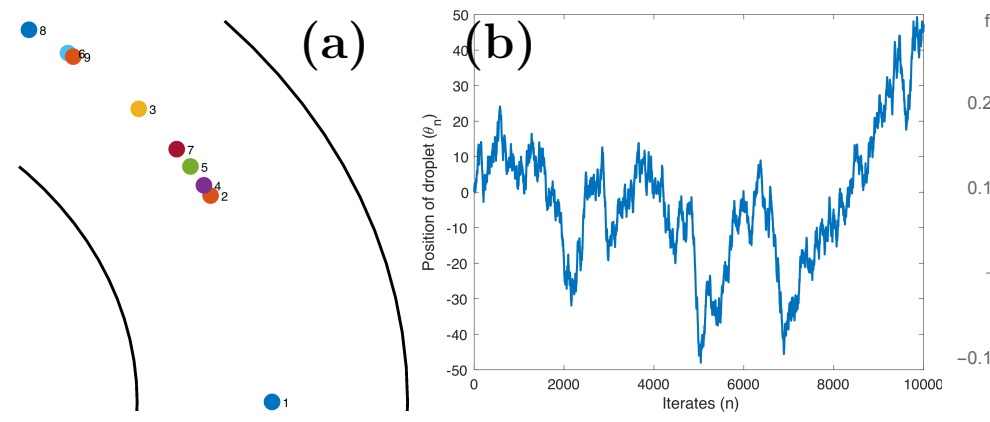

"(c)

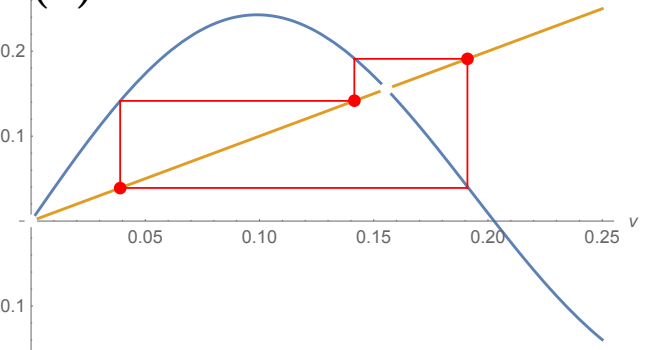

Figure 5. Chaos in the model of walkers on an annulus (17)[75]. (a) Short time dynamics of the droplets showing sudden changes in velocity. (b) Long time dynamics showing seemingly random behavior. (c) Cobweb plot of a sample 3 -cycle in the map (18).

good agreement with corresponding quantum (Schrödinger equation) results, especially for the chaotic regimes.

6.2. Some conjectures. Based on what we know of the state-of-the-art of walking-droplet dynamics research as outlined above and the current development of modern dynamical systems theory, it is reasonable to posit the following conjectures:

(C1) There is a class of smooth IDE systems including (4) that can be proved to exhibit pitchfork bifurcations, period-doubling cascades to chaos and chaotic attractors generated by interactions of invariant manifolds and possibly inertial manifolds containing global chaotic strange attractors.

(C2) It can be proved that the discrete dynamical system (16) exhibits analogs of all or most of the dynamical features in (C1). In particular, a global chaotic strange attactor is created by the decreasing distance between an expanding invariant torus and the stable manifold of a fixed point as $C \rightarrow 1$.

(C3) Informed by and building upon the work of Couder and Fort [7], Blanchette [39], Dubertrand et al. [98], Faria [61] and Nachbin et al. [99] it should be possible to construct an IDE model of walking droplet dynamics for a wide range of topographies with two very useful features: It is amenable to rigorous dynamical system analysis that reveals numerous important dynamical features such as those in $(\mathrm{C} 1)$ and $(\mathrm{C} 2)$; and its dynamical predictions are in good agreement with experiments and reliable simulations of other models.

(C4) The results in Gilet $[72,73]$ can be leveraged, mainly through the choice of eigenmodes, to find discrete dynamical analogs of (15) and (16) for variable topographies capable of producing provable dynamics analogous to those in (C3), while also exhibiting reasonably good agreement with experiments and simulations.

\section{Unsolved Problems}

In addition to the open problems associated with the conjectures $(\mathrm{C} 1)$ - $(\mathrm{C} 4)$ above, we describe two more. One is the problem of developing a generalized pilot-wave framework along the lines described in such investigations as Bush [8] and Fort and Couder [100] (see also Turton et al. [86]). 
The other is generalizing (4) to an IDE for multiple droplets that reliably predicts behavior and is amenable to dynamical systems analysis capable of proving the existence of bifurcations and chaotic regimes and possible chaotic strange attractors. In this effort, the pioneering work such as that of Borghesi et al. [101], Oza et al. [102] and Galeano-Rios et al. [103] should prove very helpful. Also, there is the related problem of extending (15) and (16) to include multiple droplet dynamics.

Reduced Dynamical Systems models can also be used to significantly decrease computational expense of creating simulations. For example, the models of Moláček and Bush [33, 34] describe the physics of walking droplets in exquisite detail, which makes it computationally prohibitive to simulate the models long enough to observe the statistical behavior. A reduced model, such as that of Oza et al. [55], is computationally fast enough to simulate on a laptop computer.

At the other end of the computational complexity spectrum, the simplified models of Gilet [72, 73], Filoux et al. [51], and Rahman and Blackmore [95, 75, 94], have very fast computation times with fine-grained statistical data from the trajectories. However, some of the physical accuracy has to be sacrificed, and while the models perform well for certain parameter regimes or a particular setup, they may miss some nuanced aspects of the phenomena. Another challenge in this regard is to build models that retain their computational speed, but include enough of the physics details to precisely capture the statistical behavior shown in experiments.

\section{ACKNOWLEDGMENTS}

The authors would like to thank A. U. Oza, J. W. M. Bush, and D. Harris for fruitful discussions. A special thank you to D. Harris for Figs. 1 and 2. A. R. appreciates the support of the Department of Applied Mathematics at UW, and D. B. appreciates the support of the Department of Mathematical Sciences at NJIT.

\section{REFERENCES}

[1] I. Newton, Philosophice Naturalis Principia Mathematica (The Royal Society, 1687).

[2] J. H. Poincaré, Les méthodes nouvelles de la mécanique céleste, volume 1 (Gauthier-Villars, Paris, France, 1892-1899).

[3] J. H. Poincaré, Leçons de Mécanique Céleste, volume 1 (Gauthier-Villars, 1905-1910).

[4] P. J. Holmes, J. Sound Vib. 84 (1982) 173.

[5] J. Walker, Sci. Am. 238 (1978) 151.

[6] Y. Couder, S. Protiere, E. Fort and A. Boudaoud, Nature 437 (2005) 208.

[7] Y. Couder and E. Fort, Phys. Rev. Lett. 97 (2006) 154101.

[8] J. W. M. Bush, Ann. Rev. Fluid Mech. 49 (2015) 269.

[9] J. W. M. Bush, Physics Today 68 (2015) 47.

[10] J. W. M. Bush, Y. Couder, T. Gilet, P. A. Milewski and A. Nachbin, Chaos 28 (2018) 096001.

[11] J. Bub, Interpreting the quantum world (Cambridge University Press, 1997).

[12] J. Bricmont, Making sense of quantum mechanics (Springer, 2016).

[13] L. de Broglie, Ondes et mouvements (Gauthier-Villars, Paris, France, 1926), 1st edition.

[14] L. de Broglie, Comptes Rendus 177 (1923) 507.

[15] L. de Broglie, Gautier-Villars, Paris .

[16] L. de Broglie, Annales de la Fondation Louis de Broglie 12 (1987) 1.

[17] D. Bohm, Phys. Rev. 85 (1952) 66.

[18] D. Bohm, Phys. Rev. 85 (1952) 180.

[19] E. Nelson, Journal of Physics: Conference Series 361 (1966) 012011.

[20] E. Nelson, Phys. Rev. 150 (1966) 1079. 
[21] Y. Couder and E. Fort, J. Phys.: Conf. Ser. 361 (2012) 012001.

[22] J. W. M. Bush and A. U. Oza, Reports on Progress in Physics (to appear).

[23] D. M. Harris and J. W. M. Bush, Phys. Fluids 25 (2013) 091112.

[24] D. M. Harris, J. Moukhtar, E. Fort, Y. Couder and J. W. M. Bush, Phys. Rev. E 88 (2013) 011001.

[25] Y. Couder, E. Fort, C.-H. Gautier and A. Boudaoud, Phys. Rev. Lett. 94 (2005) 177801.

[26] S. Protiere, A. Boudaoud and Y. Couder, J. Fluid Mech. 554 (2006) 85.

[27] T. Gilet, K. Mulleners, J. P. Lecomet, N. Vandewalle and S. Dorbolo, Phys. Rev. E 75 (2007) 36303.

[28] D. M. Harris and J. W. M. Bush, J. Sound Vib. 344 (2015) 255.

[29] D. M. Harris, T. Liu and J. W. M. Bush, Experimetns in Fluids 56 (2015) 83.

[30] G. Pucci, D. M. Harris, L. M. Faria and J. W. M. Bush, J. Fluid Mech. 835 (2018) 1136.

[31] P. Sáenz, T. Cristea-Platon and J. W. M. Bush, Nature Physics 14 (2018) 315.

[32] S. Protiere, Y. Couder, E. Fort and A. Boudaoud, J. Phys.: Condens. Matter 17 (2005) 3529.

[33] J. Molacek and J. W. M. Bush, J. Fluid Mech. 727 (2013) 582.

[34] J. Molacek and J. W. M. Bush, J. Fluid Mech. 727 (2013) 612.

[35] O. Wind-Willassen, J. Molacek, D. M. Harris and J. W. M. Bush, Phys. Fluids 25 (2013) 1.

[36] T. Gilet, N. Vandewalle and S. Dorbolo, Phys. Rev. E 76 (2007) 35302.

[37] M. Faraday, Philos. Trans. R. Soc. London 121 (1831) 319.

[38] D. Terwagne, F. Ludewig, N. Vandewalle and S. Dorbolo, Phys. Fluids 25 (2013) 122101.

[39] F. Blanchette, Phys. Fluids 28 (2016) 032104.

[40] E. Fort, A. Eddi, A. Boudaoud, J. Moukhtar and Y. Couder, Proc. Nat. Acad. Sci. 107 (2010) 17515.

[41] D. M. Harris and J. W. M. Bush, J. Fluid Mech. 739 (2014) 444.

[42] A. Oza, D. M. Harris, R. R. Rosales and J. W. M. Bush, J. Fluid Mech. 744 (2014) 404.

[43] A. Oza, O. Wind-Willassen, D. M. Harris, R. R. Rosales and J. W. M. Bush, Phys. Fluids 26 (2014) 082101.

[44] A. Oza, R. R. Rosales and J. W. M. Bush, Chaos 28 (2018) 096106.

[45] A. Eddi, J. Moukhtar, S. Perrard, E. Fort and Y. Couder, Phys. Rev. Lett. 108 (2012) 264503.

[46] M. F. Crommie, C. P. Lutz and D. M. Eigler, Science 262 (1993) 218.

[47] T. Cristea-Platon, P. Sáenz and J. W. M. Bush, Chaos 28 (2018) 096116.

[48] P. Sáenz, T. Cristea-Platon and J. W. M. Bush, Sci. Adv. 6 (2020) eaay9234.

[49] G. Pucci, P. Sáenz, L. M. Faria and J. W. M. Bush, J. Fluid Mech. 804 (2016) R3.

[50] G. Pucci, L. M. Faria, B. Aubin, P. T. Brun and J. W. M. Bush, (submitted) .

[51] B. Filoux, M. Hubert and N. Vandewalle, Phys. Rev. E 92 (2015) 041004(R).

[52] B. Filoux, M. Hubert, P. Schlagheck and N. Vandewalle, Phys. Rev. F 2 (2017) 013601.

[53] T. Gilet and J. W. M. Bush, J. Fluid Mech. 625 (2009) 167.

[54] T. Gilet and J. W. M. Bush, Phys. Rev. Lett. 102 (2009) 014501.

[55] A. Oza, R. R. Rosales and J. W. M. Bush, J. Fluid Mech. 737 (2013) 552.

[56] A. Eddi, E. Sultan, J. Moukhtar, E. Fort, M. Rossi and Y. Couder, J. Fluid Mech. 674 (2011) 433.

[57] P. A. Milewski, C. A. Galeano-Rios, A. Nachbin and J. W. M. Bush, J. Fluid Mech. 778 (2015) 361.

[58] M. Durey and P. A. Milewski, J. Fluid Mech. 821 (2017) 296.

[59] M. Durey, P. A. Milewski and Z. Wang, J. Fluid Mech. (in press).

[60] C. A. Galeano-Rios, P. A. Milewski and J.-M. Vanden-Broeck, J. Fluid Mech. 826 (2017) 97.

[61] L. M. Faria, J. Fluid Mech. 811 (2017) 51.

[62] R. N. Valani, A. C. Slim and T. Simula, Phys. Rev. Lett. 123 (2019) 024503.

[63] C. A. Galeano-Rios, P. A. Milewski and J.-M. Vanden-Broeck, J. Fluid Mech. 873 (2019) 856.

[64] J. W. M. Bush, A. U. Oza and J. Molacek, J. Fluid Mech. 755 (2014) R7.

[65] M. Labousse and S. Perrard, Phys. Rev. E 90 (2014) 022913.

[66] D. Shirokoff, Chaos 23 (2013) 013115.

[67] O. Arino, M. L. Hbid and E. A. Dads, eds., Delay Differential Equations and Applications, Proceedings of the NATO Advanced Study Institute held in Marrakech, Morocco, 9-21 September 2002 (Springer, 2002).

[68] J. Robinson, Infinite-Dimensional Dynamical Systems (Cambridge University Press, Cambridge, 2001).

[69] R. Temam, Infinite-Dimensional Dynamical Systems in Mechancs and Physics (Springer-Verlag, New York, 1997), 2nd edition.

[70] M. Durey, (submitted) . 
[71] W. Press, S. Teukolsky, W. Vetterling and B. Flannery, Numerical Recipes (Cambridge University Press, Cambridge, 2007), 3rd edition.

[72] T. Gilet, Phys. Rev. E 90 (2014) 052917.

[73] T. Gilet, Phys. Rev. E 93 (2016) 042202.

[74] A. Rahman and D. Blackmore, Chaos, Solitons \& Fractals 91 (2016) 339.

[75] A. Rahman, Chaos 28 (2018) 096102.

[76] B. V. Chirikov, Preprint N 267, Institute of Nuclear Physics, Novosibirsk (in Russian) [Engl. Transl., CERN Trans. 71 - 40, Geneva, October (1971), Translated by A. T. Sanders]. .

[77] B. V. Chirikov, Phys. Rep. 52 (1979) 263.

[78] E. Ott, Chaos in Dynamical Systems (Cambridge University Press, New York, 2002).

[79] D. K. Arrowsmith and C. M. Place, Dynamical Systems: Differential Equations, Maps and Chaotic Behaviour (Chapman and Hall, London, 1992).

[80] S. Newhouse, Topology 13 (1974) 9.

[81] J. Palis and W. de Melo, Geometric Theory of Dynamical Systems: An Introduction (Springer-Verlag, New York, 1982).

[82] C. Robinson, Dynamical Systems: Stability, Symbolic Dynamics, and Chaos (CRC Press Inc., Boca Raton, 1995).

[83] S. Smale, The Mathematics of Time: Essays on Dynamical Systems, Economic Processes, and Related Topics (Springer-Verlag, New York, 1980).

[84] S. Wiggins, Introduction to Applied Nonlinear Dynamical Systems and Chaos (Springer, New York, 2003).

[85] S. Perrard, M. Labousse, M. Miskin, E. Fort and Y. Couder, Nature Comm. 5 (2014) 3219.

[86] S. Turton, M. Couchman and J. W. M. Bush, Chaos 28 (2018) 096111.

[87] L. Tambasco, D. M. Harris, A. Oza, R. R. Rosales and J. W. M. Bush, Chaos 26 (2016) 103107.

[88] S. Newhouse, D. Ruelle and F. Takens, Commun. Math. Phys. 64 (1978) 35.

[89] K. Kurianski, A. U. Oza and J. W. M. Bush, Phys. Rev. Fluids 2 (2017) 113602.

[90] S. Perrard and M. Labousse, Chaos 28 (2018) 096109.

[91] N. Budanur and M. Fleury, Chaos 29 (2019) 013122.

[92] J. Neimark, Dokl. Akad. Nauk SSSR 129 (1959) 736.

[93] R. Sacker, Report IMM-NYU 333 (1964) 1.

[94] A. Rahman and D. Blackmore, Commun. Nonlinear Sci. Numer. Simulat. 90 (2020) 105348.

[95] A. Rahman, Y. Joshi and D. Blackmore, Regul. Chaotic Dyn. 22 (2017) 740.

[96] Y. Kuznetsov, Elements of Applied Bifurcation Theory, volume 112 (Springer-Verlag, New York, NY, 1995), 3rd edition.

[97] T. Li and J. Yorke, The American Mathematical Monthly 82 (1975) 985.

[98] R. Dubertrand, M. Hubert, P. Schlagheck, N. Vandewalle, T. Bastin and J. Martin, New J. Phys. 18 (2016) 113037.

[99] A. Nachbin, P. A. Milewski and J. W. M. Bush, Phys. Rev. F 2 (2017) 011001.

[100] E. Fort and Y. Couder, Europhys. Lett. 102 (2013) 16005.

[101] C. Borghesi, J. Moukhtar, M. Labousse, A. Eddi, E. Fort and Y. Couder, Phys. Rev. E 90 (2014) 063017.

[102] A. U. Oza, E. Siéfert, D. M. Harris, J. Molacek and J. W. M. Bush, Phys. Rev. F 2 (2017) 053601.

[103] C. A. Galeano-Rios, M. Couchman, P. Caldairou and J. W. M. Bush, Chaos 28 (2018) 096112. 